\title{
Actividades motivacionales que favorecen el incremento de la producción empresarial
}

\author{
Motivational activities that favor the increase in business production
}

\begin{abstract}
Atividades motivacionais que favorecem o aumento da produção
empresarial

Cesar Augusto Giraldo Duque

Universidad de Cundinamarca, Colombia

Cesaragiraldo1@outlook.com

Cesaragiraldo@ucundinamarca.edu.co

https://orcid.org/0000-0002-8207-2942
\end{abstract}

\section{Resumen}

Esta investigación se desarrolló en la ciudad de Bogotá y en el Departamento de Cundinamarca (Colombia) con el fin de identificar los factores que determinan la motivación y satisfacción laboral en empresas que presentan baja producción y asociatividad en los trabajadores, para lo cual se implementaron las teorías de motivación, satisfacción laboral y clima organizacional. Para conocer los problemas internos de forma individualizada se utilizó el estudio del caso, aunque también se implementó el método de investigación-acción para producir cambios en la realidad estudiada. Entre los resultados más relevantes se observó la modificación de la conducta de los jefes directos, ya que buscaban el bienestar de los colaboradores; asimismo, se apreció un acercamiento entre las directivas y el personal operativo, y se mejoró el clima organizacional. Todo esto generó mayor empatía y agrado en el desempeño de las actividades, lo que se tradujo en un incremento en la producción, así como en una mejora en la calidad de los productos y prestación de servicios por parte de los trabajadores.

Palabras clave: mano de obra, satisfacción en el trabajo. 


\section{Abstract}

This research was carried out in the city of Bogotá and in the Department of Cundinamarca (Colombia) with the purpose of identifying the factors that determine motivation and job satisfaction in companies with low production and associativity among workers, for which the theories of motivation, job satisfaction and organizational climate were implemented. In order to know the internal problems in an individualized way, the case study was used, although the action-research method was also implemented to produce changes in the studied reality. Among the most relevant results was the modification of the behavior of the direct bosses, since they sought the well-being of the collaborators; likewise, a rapprochement was observed between the managers and the operative personnel, and the organizational climate was improved. All this generated greater empathy and pleasure in the performance of activities, which resulted in an increase in production, as well as an improvement in the quality of products and services provided by the workers.

Keywords: labor, job satisfaction.

\section{Resumo}

Esta pesquisa foi desenvolvida na cidade de Bogotá e no Departamento de Cundinamarca (Colômbia) com o objetivo de identificar os fatores que determinam a motivação e a satisfação no trabalho em empresas que apresentam baixa produção e associatividade nos trabalhadores, para os quais as teorias da motivação, satisfação no trabalho e clima organizacional. Para conhecer os problemas internos individualmente, foi utilizado o estudo de caso, embora o método da pesquisa-ação também tenha sido implementado para produzir mudanças na realidade estudada. Entre os resultados mais relevantes estava a modificação do comportamento dos gestores diretos, uma vez que buscavam o bem-estar dos colaboradores; Da mesma forma, apreciou-se a aproximação entre as diretrizes e o pessoal operacional e melhorou o clima organizacional. Tudo isso gerou maior empatia e prazer no desempenho das atividades, o que resultou no aumento da produção, bem como na melhoria da qualidade dos produtos e da prestação de serviços pelos trabalhadores.

Palavras-chave: força de trabalho, satisfação no trabalho.

Fecha Recepción: Marzo 2020 Fecha Aceptación: Diciembre 2020 


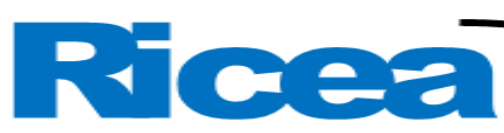

REVISTA IBEROAMERICANA DE CONTADURÍA, ECONOMÍA Y ADMINISTRACIÓN

\section{Introducción}

En las micro, pequeñas y medianas empresas (mipymes) es común que, sin determinar las casusa, los trabajadores sean retirados de sus labores cuando no cumplen con las actividades asignadas; esto decisión es tomada, generalmente, porque en dichos lugares de trabajo no existe un departamento de recursos humanos o de psicología que pueda ayudar a conocer las causas que pudieron haber generado ese incumplimiento laboral, como por ejemplo el agotamiento físico, la saturación mental, el maltrato por colegas o clientes, etc. Por eso, se hace necesario diagnosticar los problemas que pudieran existir en el interior de las organizaciones y proponer alternativas de cambio por medio de la implementación de estímulos, los cuales podrían crear un entorno tranquilo que fomente el buen desempeño de todos los funcionarios de la empresa.

En concordancia con lo anterior, en el presente trabajo se ha planteado la siguiente hipótesis: es posible incrementar la producción y la calidad en los productos y servicios de una organización después de exponer a los trabajadores a un experimento teórico-práctico en el que se utilizan las teorías de motivación, satisfacción laboral y clima organizacional como base argumentativa del ejercicio investigativo.

En la búsqueda y selección de empresas para esta investigación, se tomó en cuenta que estas no contaran con un departamento de recursos humanos, por lo que su administración debía estar centralizada en la alta dirección. Asimismo, para el desarrollo de la actividad se identificaron problemas internos en diversas organizaciones, como baja producción, problemas de asociatividad, mala calidad o mal servicio al público; luego, con base en las teorías de motivación, satisfacción laboral y clima organizacional, se realizaron acercamientos y actividades experimentales con el fin de generar cambios en la conducta laboral de las personas involucradas.

Esta investigación se ha venido realizando desde el año 2015 con los estudiantes de cuarto semestre de la Universidad de Cundinamarca; sin embargo, fue hasta el año de 2018 cuando se empezó a documentar la información en un canal de Youtube, por lo que solo se han tenido en cuenta las 45 empresas estudiadas durante el periodo 2018-2019. El trabajo de campo ha sido realizado por 199 estudiantes, y ha contado con la participación de 451 trabajadores de las referidas empresas, los cuales fueron seleccionados mediante un muestreo probabilístico, ya que todos los integrantes de la población tienen la misma posibilidad de ser elegidos (Hernández, Fernández y Baptista, 2014). 


\section{Método}

Esta investigación se concretó mediante la implementación de un estudio intrínseco de caso, el cual ofrece una mayor comprensión de los fenómenos observados. En un acercamiento inicial con las directivas de las 45 organizaciones se pudo determinar la problemática de cada una de estas.

Saltos, Loor y Palma (2018), por otra parte, plantean que la investigación-acción es participativa, ya que los investigadores trabajan con sus propias práticas incluyendo un proceso sistemático de aprendizaje, el cual se realiza con pequeños ciclos de planificación, observación y reflexión de los problemas. Mancilla, Soler y Morrón (2018) indican que esta metodología tiene un modelo crítico, cuya finalidad es la transformación social para plantear solución a los problemas mediante la observación y el análisis. Asimismo, con el método experimental se abordaron los problemas de los 451 trabajadores por medio de una manipulación deliberada de resultados que permitió observar los posibles efectos de los experimentos realizados (Hernández et al., 2014).

Siguiendo a Otero (2018), en el análisis de la información se implementó un enfoque cuantitativo que utilizó métodos científicos como teorías e hipótesis para desarrollar instrumentos de medición y manipulación de variables, así como un enfoque cualitativo (Sandoval, s. f.) que trató con fenómenos difíciles o imposibles de cuantificar matemáticamente. De este modo, se recogió información subjetiva referida a los sentimientos y valores personales y culturales de los entrevistados (Behar, 2008).

En cuanto a la colaboración de los estudiantes, estos —al ingresar al semestre académico - debían empezar la búsqueda de las empresas. En concreto, en la semana de estudio número 5 realizaban los primeros acercamientos a los trabajadores de las diferentes organizaciones, y en la semana 9 entregaban los resultados finales recabados. Paralelamente a este trabajo, se realizó una encuesta de satisfacción, las cuales fueron aplicadas en las siguientes fechas: mayo de 2018, noviembre de 2018, mayo de 2019 y noviembre de 2019 (las fechas de entrevistas se observan en la tabla 1). Esto permitió reunir información sobre las percepciones de los directivos y jefes directos, así como de los propios trabajadores sobre la vivencia en la realización de este experimento. Al final, cada grupo de estudiantes presentaba los principales hallazgos conseguidos en las empresas por medio de un video publicado

en

Youtube 


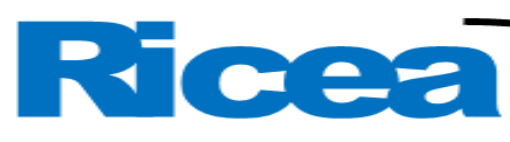

REVISTA IBEROAMERICANA DE CONTADURÍA, ECONOMÍA Y ADMINISTRACIÓN

ISSN= $2007-9907$

(https://www.youtube.com/channel/UCmohWLfOm5uJ4D7Uy4q0Brg?view_as=subscriber ) .

Vale acotar que este tipo de iniciativas son difíciles de concretar porque en muchos establecimientos exigían a los estudiantes una carta de autorización de la universidad, la cual no se pudo conseguir porque la actividad propuesta formaba parte de un trabajo de aula. Asimismo, algunas organizaciones permitieron realizar el estudio e implementar las teorías para presentarlas como actividad de aula, pero no autorizaron la divulgación de los videos en el canal de Youtube ni en ningún medio, por lo que no forman parte de este documento.

Tabla 1. Relación de fechas de encuesta con estudiantes y empresas

\begin{tabular}{|c|c|c|c|}
\hline $\begin{array}{c}\text { Fecha de encuesta a } \\
\text { estudiantes }\end{array}$ & $\begin{array}{l}\text { Estudiantes } \\
\text { involucrados }\end{array}$ & Empresas & $\begin{array}{c}\text { Empresas que no } \\
\text { autorizaron la } \\
\text { divulgación de } \\
\text { resultados }\end{array}$ \\
\hline 2018 mayo & 49 & 11 & 2 \\
\hline 2018 noviembre & 53 & 12 & 4 \\
\hline 2019 mayo & 57 & 11 & 3 \\
\hline 2019 noviembre & 40 & 11 & 2 \\
\hline Total & 199 & 45 & 11 \\
\hline
\end{tabular}

Fuente: Elaboración propia

\section{Resultados}

El estudio se concretó en el área de la administración y las organizaciones, en la línea de investigación de desarrollo organizacional y regional, lo que permitió analizar las teorías de la motivación laboral y satisfacción laboral, así como las teorías de clima organizacional, las cuales se relacionan en la siguiente tabla para su mayor comprensión y análisis. 
Tabla 2. Autores de las teorías de la motivación, satisfacción y clima organizacional

\begin{tabular}{|c|c|c|}
\hline $\begin{array}{c}\text { Teorías de la motivación } \\
\text { laboral }\end{array}$ & $\begin{array}{c}\text { Teorías de la satisfacción } \\
\text { laboral }\end{array}$ & $\begin{array}{c}\text { Teorías del clima } \\
\text { organizacional }\end{array}$ \\
\hline $\begin{array}{l}\text { Planteamientos de } \\
\text { motivación laboral de } \\
\text { Frederick Herzberg (1959). }\end{array}$ & $\begin{array}{l}\text { Los planteamientos de } \\
\text { Munsterberg (1913). }\end{array}$ & $\begin{array}{lr}\text { Dimensiones del clima } \\
\text { organizacional } & \text { de } \\
\text { Goncalves (1997). } & \end{array}$ \\
\hline $\begin{array}{l}\text { Teoría sobre la pirámide de } \\
\text { necesidades de Abraham } \\
\text { Maslow (1934). }\end{array}$ & $\begin{array}{l}\text { Teoría de los dos factores } \\
\text { motivación-higiene de } \\
\text { Herzberg Mausner y } \\
\text { Synderman (1959). }\end{array}$ & $\begin{array}{l}\text { Administración de los } \\
\text { recursos humanos } \\
\text { (Chiavenato, 2019). }\end{array}$ \\
\hline $\begin{array}{l}\text { Teoría sobre las tres } \\
\text { necesidades universales de } \\
\text { McClelland (1989). }\end{array}$ & $\begin{array}{l}\text { Teoría social cognitiva de } \\
\text { Albert Bandura (1980). }\end{array}$ & $\begin{array}{l}\text { Administración de los } \\
\text { recursos humanos } \\
\text { (Chiavenato, 1999). }\end{array}$ \\
\hline $\begin{array}{l}\text { Teoría de la equidad de John } \\
\text { Stancey Adams (1963). }\end{array}$ & $\begin{array}{l}\text { Las dimensiones de la } \\
\text { satisfacción laboral de Loke } \\
\text { (1976). }\end{array}$ & $\begin{array}{l}\text { Teoría del clima } \\
\text { organizacional de } \\
\text { Likert (1946). }\end{array}$ \\
\hline $\begin{array}{l}\text { Teoría de los refuerzos de } \\
\text { Frederick B. Skinner(1948). }\end{array}$ & & $\begin{array}{l}\text { Modelos del desarrollo } \\
\text { organizacional de Lawrence } \\
\text { y Lorsch (1967). }\end{array}$ \\
\hline $\begin{array}{l}\text { Teoría X y Y de MacGregor } \\
\text { (1966). } \\
\text { Teoría de las expectativas de } \\
\text { Víctor Vroom (1964). }\end{array}$ & & \\
\hline $\begin{array}{l}\text { Teoría de las relaciones } \\
\text { humanas de George Eltón } \\
\text { Mayo (1930). }\end{array}$ & & \\
\hline
\end{tabular}

Fuente: Elaboración propia

Ahora bien, como se muestra en la tabla 3, las mipymes que desempeñan actividades de servicio al cliente (como restaurantes, cafeterías y venta de alimentos) constituyeron 40 $\%$ de la población estudiada (es decir, 18 establecimientos), las cuales tenía un promedio de 3 a 12 trabajadores. Asimismo, en cuanto a las entrevistas con los jefes directos, manifestaron que los problemas generados en el interior de la organización pueden ser generados por sobrecarga laboral, poco empoderamiento con la organización y mucha rotación de personal 


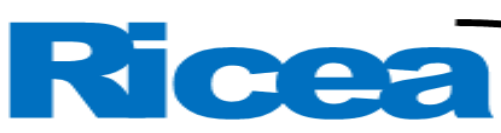

REVISTA IBEROAMERICANA DE CONTADURÍA, ECONOMÍA Y ADMINISTRACIÓN

ISSN= $2007-9907$

(por su propia voluntad). Sobre este aspecto, vale acotar que las condiciones laborales afectan directa e indirectamente en el aspecto psicosocial del trabajador, lo que incrementa el ausentismo laboral, aumenta los costos empresariales y ocasiona el desgaste del recurso humano (Jiménez, Rivera y Gaibao, 2019; Tatamuez, Domínguez, Matabanchoy, 2018).

Por otra parte, $28.89 \%$ de las mipymes elegidas (13 empresas) estaban representadas en industrias, fábricas y talleres que tenían un promedio de entre 5 y 45 trabajadores. Los problemas detectados por los jefes directos de estas organizaciones fueron bajo rendimiento y baja calidad de los productos por parte de algunos colaboradores. Las posibles causas de esta situación podrían encontrarse en las largas jornadas de trabajo de pie y la poca comunicación con los compañeros. De acuerdo con Marchena, Salazar y Vidal (2019), las empresas con mayor número de personal presentan mala comunicación entre trabajadores y directivos, lo que genera un bajo rendimiento general, mientras que Lawrence y Lorsch (citados por Mintzberg, 2012) indican que las condiciones del entorno de la organización afectan directamente en el comportamiento de los trabajadores.

Asimismo, se puede indicar que $13.33 \%$ de empresas analizadas (6 establecimientos) estaban constituidas por supermercados, ferreterías, almacenes variados y casinos que tenían un promedio de 4 a 10 trabajadores. Los problemas más significativos detectados por los jefes directos tuvieron que ver con largas jornadas de trabajo, mínimos tiempos de descanso y poco compañerismo entre colaboradores. Al respecto, Campos (30 de junio de 2019) afirma que las personas que se enfrentan a largas jornadas de trabajo tienen mayor riesgo de sufrir un accidente cerebrovascular. De hecho, los turnos irregulares, el trabajo nocturno y la tensión laboral pueden ser responsables de condiciones poco saludables a largo plazo (tabla 3).

Igualmente, $11.11 \%$ de las empresas (5 organizaciones) estuvieron conformadas por farmacias, peluquerías, parqueaderos y ventas de intangibles, las cuales contaban con un promedio de 3 a 6 trabajadores. Los problemas más significativos detectados por los jefes directos que afectan a la producción fueron las largas jornadas de trabajo y los descansos, que se realizan cada 15 días.

Por otra parte, $4.44 \%$ de las empresas (2 mypimes) contaban con un promedio de 7 a 20 trabajadores. Los problemas más significativos detectados por los jefes directos tenían que ver con el estrés y el cansancio físico, aunque también señalaron que existe compañerismo en medio de un ambiente laboral altamente competitivo. Al respecto, Jeffrey Pfeffer — profesor de la Universidad de Stanford — afirma que el exceso de trabajo está 


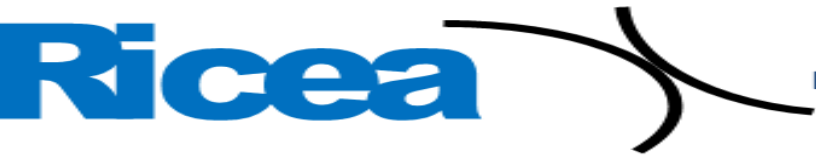

matando a las personas. De hecho, en su investigación observa que el estrés ha enfermado a $61 \%$ de los empleados de Estados Unidos, mientras que $7 \%$ han sido hospitalizados, lo que a su vez se relaciona con la muerte de un aproximado de 120000 personas anualmente, con un costo para las empresas de más de 300 millones de dólares al año (Barria, 22 de marzo de 2019).

Finalmente, $2.22 \%$ de las empresas estudiadas estuvo constituida por una entidad pública que tenía 15 funcionarios administrativos. Los problemas más significativos detectados por el jefe directo — y que afectan a la prestación del servicio — son el estrés, el aburrimiento y la monotonía, lo que genera que algunos colaboradores lleguen tarde a ejercer sus actividades y, por tanto, demora los procesos. 


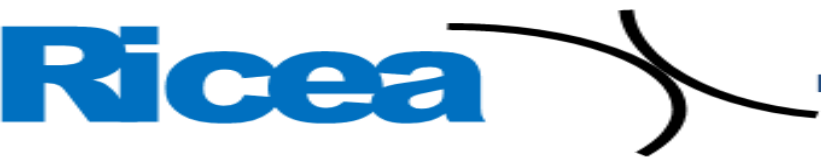

REVISTA IBEROAMERICANA DE CONTADURÍA, ECONOMIIA

YADMINISTRACIÓN

Tabla 3. Tipos de empresas estudiadas

\begin{tabular}{|c|c|c|c|c|}
\hline Tipo de empresa & Cantidad & Porcentaje & $\begin{array}{l}\text { Cantidad de } \\
\text { trabajadores }\end{array}$ & $\begin{array}{c}\text { Problemas } \\
\text { comunes }\end{array}$ \\
\hline $\begin{array}{l}\text { Restaurante, cafeterías servicios } \\
\text { de alimentos }\end{array}$ & 18 & $40.00 \%$ & 3 a 12 & $\begin{array}{l}\text { Sobrecarga } \\
\text { laboral, poco } \\
\text { empoderamiento, } \\
\text { rotación de } \\
\text { personal. }\end{array}$ \\
\hline Fábricas e industrias & 13 & $28.89 \%$ & 5 a 45 & $\begin{array}{l}\text { Largas jornadas } \\
\text { de trabajo de pie } \\
\text { y poca } \\
\text { comunicación } \\
\text { con los } \\
\text { compañeros. }\end{array}$ \\
\hline $\begin{array}{l}\text { Supermercados, almacenes, } \\
\text { ferreterías y casinos }\end{array}$ & 6 & $13.33 \%$ & 4 a 10 & $\begin{array}{l}\text { Largas jornadas } \\
\text { de trabajo, } \\
\text { mínimos tiempos } \\
\text { de descanso y } \\
\text { poco } \\
\text { compañerismo } \\
\text { entre } \\
\text { colaboradores. }\end{array}$ \\
\hline $\begin{array}{l}\text { Farmacias, peluquerías, } \\
\text { parqueaderos y ventas de } \\
\text { intangibles }\end{array}$ & 5 & $11.11 \%$ & 3 a 6 & $\begin{array}{l}\text { Largas jornadas } \\
\text { de trabajo, un } \\
\text { descanso } \\
\text { quincenal. }\end{array}$ \\
\hline $\begin{array}{l}\text { Empresas de asesorías } \\
\text { profesionales }\end{array}$ & 2 & $4.44 \%$ & 7 a 20 & $\begin{array}{l}\text { Estrés y } \\
\text { cansancio físico. }\end{array}$ \\
\hline Entidad pública & 1 & $2.22 \%$ & 15 & $\begin{array}{l}\text { Estrés, } \\
\text { aburrimiento y la } \\
\text { monotonía. }\end{array}$ \\
\hline
\end{tabular}

Fuente: Elaboración propia 


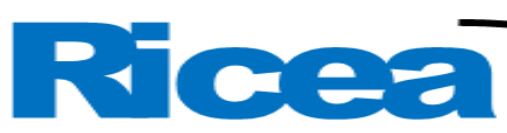

REVISTA IBEROAMERICANA DE CONTADURÍA, ECONOMÍA Y ADMINISTRACIÓN

ISSN $=2007-9907$

Por otra parte, en la tabla 4 se observa que $21.11 \%$ de los trabajadores manifestaron que tenían sobrecarga laboral, no disponían de hora de salida y muy pocas veces se les reconocían las horas extras. De hecho, en algunas organizaciones se descansa cada 15 días, ya que no existe personal para realizar los reemplazos.

Asimismo, $12.56 \%$ de trabajadores laboraba sin ningún tipo de motivación, lo que se relaciona con una baja autoestima y resentimiento con los compañeros y jefes directos. Igualmente, $11.06 \%$ manifestaba bajo rendimiento laboral porque se sentían descuidados por la alta dirección. De acuerdo con Rodríguez (2020), la motivación del personal en la empresa tiene relación directa con la efectividad y el compromiso organizacional, y la rotación laboral puede generar bajo rendimiento en el trabajo (Campos, Gutiérrez y Matzumura, 2020).

Además, $10.5 \%$ de los colaboradores indicaron que tenían poco compromiso laboral, y ese mismo porcentaje consideró que las actividades desempeñadas no se ajustaba a sus perfiles ni a sus necesidades, por lo que se limitan a cumplir con lo asignado. Es en este tipo de situaciones se identifica la teoría X y Y de MacGregor (citado por Martín, 13 de julio de 2017), donde $Y$ está representado por personas trabajadoras y positivas, mientras que X son aquellas que se aprovechan de la situación, no tienen compromiso y nunca se sienten satisfechas con las actividades. Para evitar este tipo de inconvenientes es necesario implementar los planteamientos de Munsterberg (citado por Burgos, Anaya, Núñez y Castro, 2018), quien indica que la satisfacción laboral es importante, ya que permite comprender el comportamiento de los trabajadores y evidenciar la personalidad de cada individuo.

Entre los problemas con menor porcentaje se encuentran la poca integración entre los trabajadores $(8.54 \%)$, tener más de un jefe directo $(7.14 \%)$ y el desconocimiento de funciones (4.02\%). Según Chávez (2018), las organizaciones exitosas se enfocan más en el recurso humano haciendo énfasis en las capacidades de los trabajadores, por lo que se deben utilizar métodos para el desarrollo de la competencia dentro del trabajo. 


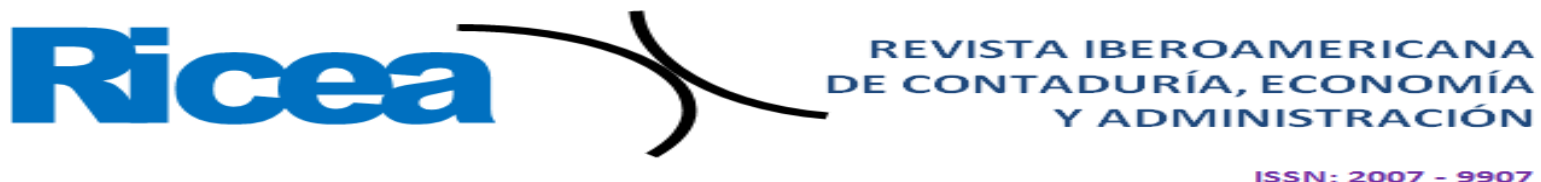

Tabla 4. Problemas comunes en la organización según la visión de los trabajadores

\begin{tabular}{|l|r|c|}
\hline \multicolumn{1}{|c|}{$\begin{array}{c}\text { Problemas más comunes de los } \\
\text { trabajadores en su labor }\end{array}$} & Porcentaje & $\begin{array}{c}\text { Cantidad de } \\
\text { trabajadores }\end{array}$ \\
Sobrecarga laboral & $21.11 \%$ & 42 \\
Poca motivación laboral & $12.56 \%$ & 25 \\
Bajo rendimiento & $11.06 \%$ & 22 \\
Conformismo laboral & $10.05 \%$ & 20 \\
Falta de compromiso & $10.05 \%$ & 20 \\
Poca integración laboral & $8.54 \%$ & 17 \\
Más de un jefe directo & $7.54 \%$ & 15 \\
Desconocimiento de funciones & $4.02 \%$ & 8 \\
Malas condiciones en el puesto & $3.02 \%$ & 6 \\
laboral & & \\
Poco respeto entre compañeros & $3.02 \%$ & 6 \\
No hay elementos de protección & $3.02 \%$ & 6 \\
Cansancio y aburrimiento & $3.02 \%$ & 6 \\
Poca comunicación con las & $3.02 \%$ & 6 \\
directivas & & \\
\hline
\end{tabular}

Fuente: Elaboración propia

Ahora bien, las actividades motivacionales, de satisfacción laboral y clima organizacional se implementaron en las diferentes organizaciones según los problemas detectados por los jefes directos, así como por los trabajadores. En tal sentido, y como se observa en la tabla 5 , a $15.56 \%$ de la población que durante toda su jornada laboral permanecía de pie o sentados se les ofrecieron pausas activas y descansos a determinadas horas. Esto generó el cambio de actitud de las personas, ya que sonreían, conversaban entre ellas y se percibía mucho compañerismo. Este ejercicio provocó un incremento en la producción sin tener que realizar indicaciones al respecto. La actividad se realizó teniendo en cuenta los aportes de la teoría de Mausner y Synderman (citados por Mancilla, Galarza y Zambrano, 2019), los cuales indican que las personas pasan por varias etapas para procurar lograr una relación equilibrada entre necesidades, capacidades, motivaciones y oportunidades. Asimismo, se tuvo en cuenta la teoría de Maslow (citado por Castro, 2018), quien plantea que el ser humano necesita suplir sus necesidades básicas y se esfuerza para lograrlo, mientras que Skinner (1948) indica que los estímulos actúan directamente en el 


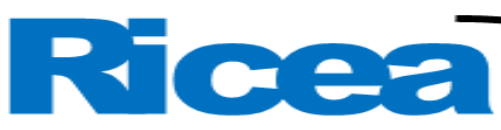

REVISTA IBEROAMERICANA DE CONTADURÍA, ECONOMÍA Y ADMINISTRACIÓN

ISSN= $2007-9907$

comportamiento de las personas. Además, Mayo (citado por Mendoza, 2018) señala que el incremento de la producción se debe a la motivación e interrelación con los equipos de trabajo.

Por otra parte, a otro $15.56 \%$ de los trabajadores tuvieron la oportunidad de realizar celebraciones de cumpleaños, día de la amistad, Hallowen y salir de paseo, lo que generó empoderamiento con la organización y mejoró el trato con los compañeros. Asimismo, a otro $15.56 \%$ de trabajadores se les ofreció un desayuno, lo que mejoró el clima organizacional y se observó que terminaron sus labores antes de tiempo. En estas actividades se tuvieron en cuenta los planteamientos de Herzberg (citado por Alvarado y Arguello, 2018), quien señala que los factores motivadores incrementan la producción, mientras que Bandura (citado por Salas, Vega, González, 2019) comenta que la conducta de las personas se puede derivar de su entorno y para detectarlo solo basta con analizar algunas de sus fortalezas. De acuerdo con Chiavenato (1999), el clima organizacional se mejora si existe buena relación entre administrativos y trabajadores, por lo que cualquier cambio en el entorno laboral altera la conducta de los colaboradores.

Asimismo, a otro $15.56 \%$ de los empleados que se desempeñaban en el área de servicio al cliente y ventas de productos y servicios se les ofrecieron obsequios y comisiones por el cumplimiento de objetivos de venta, mientras que a otro grupo de esta población se les brindaron clases de relajación. En este sentido, el primer grupo empezó a trabajar en un ambiente competitivo, dejaron a un lado los valores y la amistad generando confrontaciones internas por el logro de sus metas, mientras que el segundo grupo consiguió una buena comunicación, trabajaban en equipo de forma colaborativa, mejoró el servicio al cliente y se incrementó la producción. De acuerdo con Likert (1946), si los supervisores muestran más interés en los resultados, el trabajador baja su moral y el ambiente de trabajo se vuelve tenso, lo que acaba con el compañerismo porque prevalece la competencia, mientras que Stancey (citado por Giraldo, 2018) indica que las personas se enfocan en el criterio que tienen en función de las recompensas; por ello, el pago de incentivos económicos no siempre arroja un buen resultado, ya que puede crear ambientes dependientes, puesto que las personas se adaptan fácilmente a esta situación y solo dan lo mejor cuando se les ofrece una retribución (Recursos Humanos, s. f.).

Además, a otro $15.56 \%$ de los trabajadores se les ofreció capacitaciones sobre servicio al cliente, seguridad y salud en el trabajo, comportamiento y ética laboral, lo que produjo que el clima organizacional mejorara. Se observó, además, que los colaboradores se 


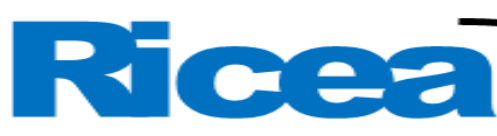

REVISTA IBEROAMERICANA DE CONTADURÍA, ECONOMÍA Y ADMINISTRACIÓN

ISSN= $2007-9907$

mostraban más cuidadosos con los productos en procura de que no se deterioraran, y al mismo tiempo los espacios estaban más aseados y bien presentados. Al respecto, Vroom (citado por Lizana, 2018) centra su teoría en el proceso de motivación en torno al trabajo, las metas y las actividades personales que mejoran el ciclo productivo, mientras que Chiavenato (2019) plantea que el trabajador pasa la mayor parte del tiempo en la organización, por lo que todas las actividades que realiza se verán reflejadas en su labor.

Por otra parte, a $11.11 \%$ de los trabajadores que tenían jornadas divididas con varias horas de intermedio y presentaron problemas de bajo rendimiento y cansancio laboral, se les ofreció un refrigerio con un descanso en cada jornada, lo que mejoró de forma inmediata el comportamiento y la producción. Para Goncalves (1997), el comportamiento de un trabajador no solo es el resultado de factores organizacionales, ya que depende de las percepciones que tiene de estos, por lo que describe el clima organizacional como una expresión de los trabajadores y directivos.

Asimismo, a $6.67 \%$ de los trabajadores se les brindaron permisos para salir a estudiar, por lo que buscaban cumplir con las metas laborales y trabajar los fines de semana para recuperar el tiempo, lo que provocó un empoderamiento con sus organizaciones.

En cuanto a $2.22 \%$ de los colaboradores que tenían descansos cada 15 días, se les propuso cumplir con los objetivos diarios de venta a cambio de un descanso por semana, lo que motivó a las personas a trabajar en equipo para alcanzar las metas propuestas. Sobre este aspecto, Locke (1976) plantea que cuando una persona tiene una meta o propósito claro, buscará cumplirlo, lo que aumentará el rendimiento laboral. De hecho, McClelland (citado por Ceolevel, 2018) sostiene que los individuos en busca de su satisfacción laboral deben suplir su necesidad de logro, la necesidad de afiliación, de poder e influencia. 
Tabla 5. Actividades motivacionales propuestas y sus resultados

\begin{tabular}{|c|c|c|c|}
\hline Actividades motivacionales & Empresas & Porcentaje & Resultado \\
\hline Descansos o pausas activas & 7 & $15.56 \%$ & $\begin{array}{l}\text { Incremento en la } \\
\text { producción sin tener que } \\
\text { realizar indicaciones al } \\
\text { respecto. }\end{array}$ \\
\hline Celebraciones en un día especial & 7 & $15.56 \%$ & $\begin{array}{l}\text { Incrementó la } \\
\text { producción }\end{array}$ \\
\hline Desayuno o refrigerios & 7 & $15.56 \%$ & $\begin{array}{l}\text { Terminaron sus labores } \\
\text { antes de tiempo }\end{array}$ \\
\hline $\begin{array}{l}\text { Obsequios por cumplimiento de } \\
\text { objetivos }\end{array}$ & 7 & $15.56 \%$ & $\begin{array}{l}\text { Trabajaban en equipo de } \\
\text { forma colaborativa y se } \\
\text { mejoró el servicio al } \\
\text { cliente y se incrementó la } \\
\text { producción. }\end{array}$ \\
\hline $\begin{array}{l}\text { Reuniones entre directivas y } \\
\text { trabajadores }\end{array}$ & 7 & $15.56 \%$ & $\begin{array}{l}\text { Los trabajadores están } \\
\text { cuidando la mercancía y } \\
\text { tienen el establecimiento } \\
\text { aseado. }\end{array}$ \\
\hline Descansos con refrigerio & 5 & $11.11 \%$ & \begin{tabular}{|lll} 
Mejoraron & el \\
comportamiento y & la \\
producción. & & \\
\end{tabular} \\
\hline Oportunidades de estudio & 3 & $6.67 \%$ & Empoderamiento laboral \\
\hline Descanso remunerado & 1 & $2.22 \%$ & $\begin{array}{l}\text { Trabajo en equipo, } \\
\text { cumplimiento de metas }\end{array}$ \\
\hline Alimentación y vivienda & 1 & $2.22 \%$ & Empoderamiento laboral \\
\hline
\end{tabular}

Fuente: Elaboración propia 


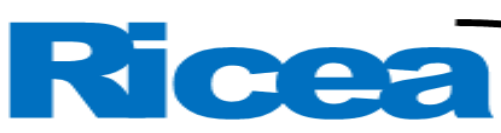

REVISTA IBEROAMERICANA DE CONTADURÍA, ECONOMÍA Y ADMINISTRACIÓN

\section{Discusión}

Esta investigación surgió en un aula de clase en el año 2015 cuando los estudiantes de la asignatura Pensamiento Administrativo manifestaban que las teorías motivacionales tenían un enfoque psicológico y que únicamente darían resultados si se utilizaban por profesionales de esta rama, por lo que para ellos resultaban inservibles en su formación de contaduría pública. Esta percepción sirvió para proponer un planteamiento de problema, el cual se enfocó en analizar los inconvenientes productivos en una empresa desde el punto de vista del supervisor; para ello, sin embargo, se tendrían que identificar los problemas en los trabajadores que originan la disminución de la producción para luego, con base en las teorías motivacionales y de satisfacción laboral, realizar propuestas para implementar actividades con los afectados sin que se dieran cuenta de que eran observados con el fin de incrementar la producción y el cambio en sus conductas laborales.

En tal sentido, los primeros resultados fueron muy positivos, tanto para los estudiantes que verificaron la veracidad de las teorías como para los empresarios que lograron incrementar la producción y mejorar el clima organizacional por medio de la utilización de pequeños estímulos que, de forma psicológica, cambiaron la forma de trabajar de los colaboradores, los cuales se mostraron empoderados con las organizaciones.

A partir de los resultados del primer ejercicio, se empezó a realizar esta actividad con los estudiantes de esta asignatura mientras se fue mejorando la estructura investigativa para diseñar un modelo de trabajo que orientara a los profesionales en formación y permitiera analizar la importancia de los resultados obtenidos. Luego, a partir del año 2018, se empezaron a documentar estas actividades en la plataforma de Youtube, lo que sirvió como material de consulta para aquellos estudiantes que iniciaban su proceso de investigación.

En tal sentido, los jefes directos, en una entrevista inicial, señalaban los problemas que existían en sus organizaciones. Asimismo, a los trabajadores u operarios se les entrevistó para identificar si tenían algún malestar con la empresa que los desmotivara. Con los datos recabados en ambas entrevistas se efectuó un cruce de información para determinar las actividades motivacionales que se podían implementar en cada una de estas empresas. En algunas empresas se realizaron entre dos y tres actividades motivacionales para analizar la conducta y los cambios detectados. Después se preguntó al supervisor o jefe, así como a los empleados si la actividad había sido positiva o no. 


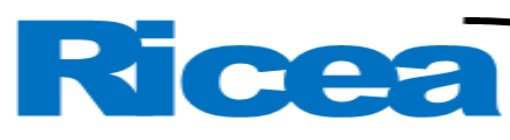

REVISTA IBEROAMERICANA DE CONTADURÍA, ECONOMÍA Y ADMINISTRACIÓN

ISSN= $2007-9907$

Entre los resultados más sobresalientes observados por los trabajadores se destacan que sus jefes modificaron su comportamiento, por lo que los escuchaban más. De hecho, ahora hacían parte del equipo de trabajo y se integraban con el personal, lo que provocaba en ellos empoderamiento con la organización. Asimismo, indicaron que el trabajo se facilitaba cuando no se ejercía presión directa.

En cuanto a los estudiantes, manifestaron que tenían nuevos conocimientos sobre el manejo de personal y trato a los trabajadores. De hecho, lo que más valoraron fue haber aprendido a generar cambios en la conducta de los colaboradores incrementando la producción sin necesidad de realizar despidos de personal, puesto que se mejora la calidad en la prestación de servicios y atención al cliente. Asimismo, se percibe que el trabajador está alegre mientras despacha pedidos, se presenta un incremento en la producción sin necesidad de ejercer presión en el trabajador, se evidencia una mejora significativa en la calidad de los productos y servicios, y disminuyen las pérdidas por productos defectuosos o daño de mercancía. En pocas palabras, se percibe que los trabajadores adquieren empoderamiento con la empresa, están más atentos a las sugerencias y se ha despertado un sentimiento de trabajo en equipo. En todas las mipymes el resultado fue $100 \%$ satisfactorio para los jefes directos y administrativos, ya que se logró cumplir con todas las expectativas propuestas.

\section{Conclusiones}

En esta investigación se logró dar cumplimiento a los objetivos propuestos, ya que se implementaron actividades que permitieron mejorar el entorno de trabajo por medio de la motivación, satisfacción y clima organizacional. De este modo se logró cambiar el comportamiento psicosocial en el interior de las organizaciones tanto en jefes directos como en operarios, lo que derivó en un ambiente sano y productivo. Además, se cumplió con la hipótesis planteada, por lo que se puede afirmar que es posible incrementar la producción y la calidad en los productos y servicios de una organización después de exponer a los trabajadores a un experimento teórico-práctico en el que se utilizan las teorías de motivación, satisfacción laboral y clima organizacional como base argumentativa del ejercicio investigativo. En efecto, las organizaciones intervenidas presentaron un cambio favorable en cuanto al manejo de personal, ya que las directivas lograron evidenciar las falencias que se 


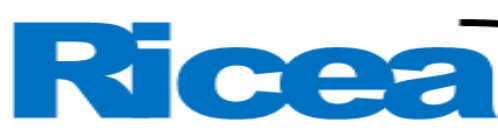

REVISTA IBEROAMERICANA DE CONTADURÍA, ECONOMÍA Y ADMINISTRACIÓN

ISSN $=2007-9907$

presentaban. Esto sirvió para que realizaran planes de trabajo a futuro para seguir creciendo de forma adecuada, brindando a sus trabajadores un trato digno.

El comportamiento de los trabajadores en el interior de las empresas es una condición que se puede manejar y moldear según las necesidades de cada entorno; solo basta con conocer las carencias de los individuos y atenderlas mediante ciertos estímulos ofrecidos a horas determinadas, en los momentos indicados.

El comportamiento de los trabajadores, tanto en Colombia como en otros países, se relaciona directamente con las culturas y costumbres sociales, ya que muchos colaboradores dan lo mejor de sí solo cuando están siendo observados y cuando reciben beneficios adicionales. Para un trabajador que se acaba de vincular a una organización es normal que los primeros consejos de sus compañeros estén dirigidos a promover un esfuerzo mínimo, ya que las empresas no suelen recompensar el rendimiento adicional. Por eso, se hace necesario que las primeras instrucciones sean realizadas únicamente por los supervisores a cargo, ya que estos están más involucrados con los propósitos y metas de la empresa.

En cuanto al clima organizacional, se conoce que los trabajadores reflejan en su comportamiento la personalidad aprendida en su entorno laboral, por lo que si este es estresante y conflictivo, no es extraño que sus colaboradores tengan esa conducta tanto en el interior de la empresa como fuera de ella. Por ende, es recomendable mantener un ambiente laboral sano que brinde estabilidad anímica y psicológica, en especial para personas que en sus hogares deben lidiar con situaciones adversas. No se debe olvidar que los individuos tienen diversas personalidades que se relacionan con sus culturas, hábitos y costumbres, por lo que actúan de una u otra manera dependiendo de los acontecimientos que deben enfrentar. 


\section{Referencias}

Alvarado, A. A. y Arguello, N. R. (2018). Factores higiénicos y motivadores en una agencia automotriz del estado de Sinaloa. Ra Ximhai, 14(3). Recuperado de https://dialnet.unirioja.es/servlet/articulo?codigo $=6916100$

Barria, C. (22 de marzo de 2019). El trabajo está matando a la gente y a nadie le importa. BBC News. Recuperado de https://www.bbc.com/mundo/noticias-47656050

Behar, D. S. (2008). Metodología de la investigación. Ediciones Shalom. Recuperado de http://rdigital.unicv.edu.cv/bitstream/123456789/106/3/Libro\%20metodologia\%20i nvestigacion\%20este.pdf

Burgos, E. C., Anaya, C. A., Núñez, M. A. y Castro, R. I. (2018). Relación y diferencia entre las dimensiones de satisfacción laboral. Revista Electrónica de Psicología Iztacala, 21(2). Recuperado de http://revistas.unam.mx/index.php/repi/article/view/65281

Campos, B. (30 de junio de 2019). Una investigación señala que largas jornadas de trabajo aumentan los riesgos de sufrir accidentes cerebrovasculares. El Cierre Digital. Recuperado de https://elcierredigital.com/salud-y-bienestar/79727845/largasjornadas-trabajo-aumentan-riesgo-accidente-cerebrovascular.html

Campos, I., Gutiérrez, H. y Matzumura, J. P. (2020). Rotación y desempeño laboral de los profesionales de enfermería en un instituto especializado. Revista Cuidarte, 10(2). Recuperado de http://www.scielo.org.co/scielo.php?script=sci_arttext\&pid=S221609732019000200202\&lang=es

Castro, F. J. (2018). Abraham Maslow, las necesidades humanas y su relación con los cuidadores profesionales. Cultura de los Cuidados, 22(52). Doi: http://dx.doi.org/10.14198/cuid.2018.52.09

Ceolevel (2018). ¿Conoces la teoría de las 3 necesidades universales de McClelland? Ceolevel. Recuperado de http://www.ceolevel.com/teoria_mcclelland

Chávez, L. E. (2018). La gestión del talento humano como estrategia empresarial. Revista Perspectiva, 19(4). Recuperado de https://revistas.upagu.edu.pe/index.php/PE/article/view/614

Chiavenato, I. (1999). Administración de recursos humanos: interacción entre personas y organizaciones (5. ${ }^{\mathrm{a}}$ ed.). Recuperado de http://www.ucipfg.com/Repositorio/MAES/MAES-08/UNIDADES- 


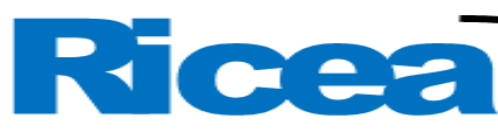

REVISTA IBEROAMERICANA DE CONTADURÍA, ECONOMÍA YADMINISTRACIÓN

APRENDIZAJE/Administracion\%20de\%20los\%20recursos\%20humanos(\%20lect \%202)\%20CHIAVENATO.pdf

Chiavenato, I. (2019). Administración de recursos humanos: el capital humano de las organizaciones (10. ${ }^{\mathrm{a}}$ ed.). Bogotá, Colombia: Mc Graw Hill.

Giraldo, L. F. (2018). Propuesta para la mejora del clima organizacional en microempresas en Colombia (trabajo de grado de especialización). Fundación Universidad de América. Recuperado

de http://repository.uamerica.edu.co/handle/20.500.11839/7085

Goncalves, A. P. (1997). Dimensiones del clima organizacional. Recuperado de http://www.geocities.ws/janethqr/liderazgo/130.html

Hernández, R., Fernández, C. y Baptista, P. (2014). Metodología de la investigación (6. ${ }^{\mathrm{a}}$ ed.). Bogotá, Colombia: McGraw-Hill Interamericana.

Jiménez, G. M., Rivera, A. M. y Gaibao, M. C. (2019). Las condiciones de bienestar laboral en una empresa del sector hotelero. Estudio de caso en Medellín, Colombia. $\begin{array}{lll}\text { Prospectiva, } & \text { (28). } & \text { Recuperado }\end{array}$ http://www.scielo.org.co/scielo.php?script=sci_arttext\&pid=S0122$12132019000200203 \&$ lang $=\mathrm{pt}$

Likert, R. (1946). Teoría del clima organizacional de Likert. Administración II, ingeniería en Transporte. Recuperado de http://administracion2transporte.blogspot.com/2009/02/teoria-del-climaorganizacional-de.html

Lizana, R. I. (2018). Propuesta de un programa de motivación basado en la teoría de la expectativa de Vroom, para el puesto de salud Horacio Zevallos, Distrito de Ate, 2018 (trabajo de grado). Universidad Peruana Unión. Recuperado de https://repositorio.upeu.edu.pe/handle/UPEU/1353

Locke, E. A. (1976). La naturaleza y las causas de la satisfacción laboral. En Dunnette, M. D. (ed.), Manual de psicología industrial y organizacional (pp. 1297-1343). Chicago, IL: Rand McNally.

Mancilla, J. A., Galarza, M. y Zambrano, D. (2019). Incidencia del capital humano de las campesinas de la comuna de Barcelona en la sostenibilidad comercial de la Paja Toquilla. Revista Empresarial, 13(2). Recuperado de https://dialnet.unirioja.es/servlet/articulo?codigo=7361514 
Mancilla, L., Soler, C. y Morrón, C. (2018). La investigación-acción en la formación del profesorado. Nuevas perspectivas desde el Proyecto Roma. Revista Iteruniversitaria de Formación del Profesorado, (92). Recuperado de https://dialnet.unirioja.es/servlet/articulo?codigo $=6627822$

Marchena, S. C., Salazar, L. F. y Vidal, Z. (2019). Implementación de un taller de control de procesos para mejorar el desempeño laboral de los colaboradores de una empresa. $D a$ Vinci Science.

Recuperado

de https://emprendeupd.com/ojs30/index.php/science/article/view/88

Martín, J. (13 de julio de 2017). ¿Conoces la teoría X y la teoría Y? Cerem. Recuperado de https://www.cerembs.co/blog/conoces-la-teoria-x-y-teoria-y

Mendoza, L. A. (2018). Plan estratégico sustentado en las teorías sociales de la administración para corregir el inapropiado clima organizacional (tesis de maestría). Universidad Nacional Pedro Ruiz Gallo. Recuperado de http://repositorio.unprg.edu.pe/handle/UNPRG/6572

Mintzberg, H. (2012). Estructuración de las organizaciones. Recuperado de https://books.google.com.co/books?id=mjOBU2PRoRoC\&pg=PA36\&dq=libro+jay +lorsch\&hl=es\&sa=X\&ved=0ahUKEwiTtYDog-HkAhUhmAKHR1nCgkQ6AEIKTAA\#v=onepage $\& \mathrm{q}=$ libro\%20jay\%20lorsch \&f=false

Otero, A. (2018). Enfoques de investigación. Métodos para el diseño urbano arquitectónico. Universidad de Atlántico. Recuperado de https://www.researchgate.net/publication/326905435_ENFOQUES_DE_INVESTI

\section{GACION}

Recursos Humanos (s. f.). Teoría de la contingencia de Lawrence y Lorsch. Los Recursos Humanos.com. Recuperado de https://www.losrecursoshumanos.com/teoria-de-lacontingencia-de-lawrence-ylorsch/

Recursos Humanos (s. f.). Teoría de la equidad de John Stacey Adams. Los Recursos Humanos. Recuperado de https://www.losrecursoshumanos.com/teoria-de-laequidad-laboral-de-stacey-adams/

Rodríguez, N. (2020). El salario emocional y su relación con los niveles de efectividad. Universidad $\quad y \quad$ Sociedad, 12(1). Recuperado de http://scielo.sld.cu/scielo.php?script=sci_arttext\&pid=S2218$36202020000100141 \&$ lang=es 
Salas, G., Vega, M., González, C., Ossa, J., Cudina, J., Rodríguez, T., Barboza, M., Ventura, J., Guerra, L. y López, W. (2019). Cincuentenario de la Revista Latinoamericana de Psicología: un análisis bibliométrico. Revisa Latinoamericana de Psicología, 51(2). Recuperado de https://dialnet.unirioja.es/servlet/articulo?codigo=7532868

Saltos, L. J, Loor, L. y Palma, M. M. (2018). La investigación acción como una estrategia pedagógica de relación entre lo académico y social. Ciencias de la Educación, 3. Recuperado de https://webcache.googleusercontent.com/search?q=cache:Jyul6p7SMWAJ:https://di alnet.unirioja.es/descarga/articulo/7183551.pdf $+\& \mathrm{~cd}=1 \& \mathrm{hl}=\mathrm{es} \& \mathrm{ct}=\mathrm{clnk} \& \mathrm{gl}=\mathrm{co}$

Sandoval, C. A. (s. f.). Investigación cualitativa. Programa de especialización en teoría, métodos y técnicas de investigación social. Instituto Colombiano para el Fomento de la Educación $\quad$ Superior ICFES. Recuperado de https://panel.inkuba.com/sites/2/archivos/manual\%20colombia\%20cualitativo.pdf

Skinner, F. B. (1948). Teoría de los refuerzos. Recuperado de https://sites.google.com/site/teoriadelreforzamiento/

Tatamuez, R. A, Domínguez, N. M. y Matabanchoy, S. M. (2018). Revisión sistemática: factores asociados al ausentismo laboral en países de América Latina. Universidad y Salud, 21(1). Recuperado de http://www.scielo.org.co/pdf/reus/v21n1/2389-7066reus-21-01-100.pdf 